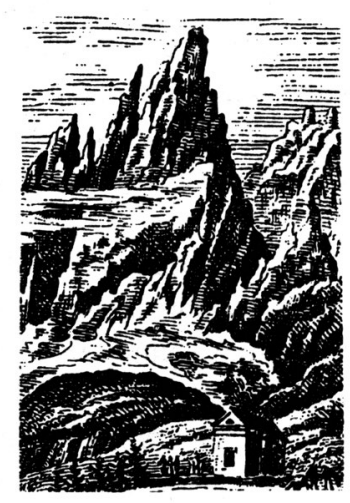

\title{
Monika Mańczyk-Krygiel
}

ORCID: 0000-0003-0588-9526

Universität Wrocław

monika.manczyk-krygiel@uwr.edu.pl

https://doi.org/10.19195/2084-4107.15.5

\section{Der Scheitel der Welt. Aline Valangin und das Onsernonetal}

Słowa-klucze: Onsernone, geopoetyka, granica, odmienność

Keywords: Onsernone, geopoetics, border, alterity

\section{The top of the world: Aline Valangin and Onserone Valley}

\section{Summary}

These considerations are devoted to literary pictures of the Onsernone Valley located on the Italian-Swiss border. It was here in the 1930s and 1940s that the Swiss writer Aline Valangin (18891986) created an extraordinary oasis of freedom and peace in her estate in Comologno. She hosted famous figures such as Kurt Tucholsky, Elias Canetti, Ignazio Silone, or Wladimir Vogel, and provided shelter to many politically persecuted artists. The subject of detailed reflection is the question of the perception, experience and acquisition of the Ticino mountains both in works by Valangin and in biographical works about her; with a particular focus on narrative perspective - from the outside and the inside.

Eveline Hasler in biographical novel Aline und die Erfindung der Liebe (2000) attempts to (re)construct an image of the Onsernone Valley as a specific "valley of poets", presenting a subtle analysis of the interaction between the conservative inhabitants of the valley, attached to tradition, 
and the extravagant artists who found asylum and inspiration in the Ticino Alps. This novel is an example of a modern biography, which is characterized by narrative polyphony; the description of space becomes an important carrier of meanings and collective memory in the author's concept.

Aline Valangin sketches in her novels (Die Bargada, 1943 / Dorf an der Grenze, 1982) and short stories (Tessiner Erzählungen, 2018) an image of Onserone indigenous people's everyday life in the thirties and forties, full of worries. Her stories include outsiders, misfits, social outcasts, guerrillas, smugglers, and exiles - and they all find haven in the Valley. Valangin's works are also an important voice in the discussion of the essence of Swiss patriotism not only through strong criticism of Swiss immigration policy during World War II, but also by reflecting on the concept of the border as a place that unexpectedly proves to be a challenge and a particular kind of self-experience in the face of events that are tearing up the current existence.

Das Tessiner Onsernonetal im schweizerisch-italienischen Grenzgebiet wird durch seine dichten Wälder, sagenhafte Schluchten und schäumende Wildwasser geprägt. Es ist rau, ursprünglich und abgelegen. In den 1930er und 1940er Jahren schuf hier die Schriftstellerin Aline Valangin (eigtl. Aline Ducommun, verh. 1) Rosenbaum, 2) Vogel; 1889-1986) eine Insel der Freiheit und des Friedens. Die ausgebildete Pianistin und Psychoanalytikerin beherbergte in ihrem Haus in Comologno so illustre Gäste wie Kurt Tucholsky, Elias Canetti, Ignazio Silone oder Wladimir Vogel und gewährte vielen politisch Verfolgten Unterschlupf. Zu ihrem Freundeskreis gehörten namhafte Künstler aus der ganzen Welt; ihre Häuser in Zürich und in Comologno galten als bedeutende literarische Salons, die den Vertretern diverser Künste Austauschmöglichkeiten gewährten. Der vorliegende Beitrag setzt sich mit der Frage nach der Erfahrung und Aneignung der Tessiner Berge in ausgewählten Werken von und über Aline Valangin auseinander, wobei unterschiedlichen Betrachtungsperspektiven - von innen versus von außen - eine besondere Aufmerksamkeit geschenkt wird.

Die Schweizer Schriftstellerin Eveline Hasler (re)konstruiert das Bild von dem Onsernonetal als einem spezifischen „Tal der Dichter” in dem biografischen Roman Aline und die Erfindung der Liebe (2000) und bietet dabei eine einfühlsame Analyse der Interaktionen zwischen den konservativen und traditionsbewussten Einheimischen und den extravaganten Künstlern, die die Tessiner Berge für sich als Zufluchtsort oder Inspirationsquelle entdeckt haben. Im Mittelpunkt des Romans stehen die im La Barca genannten Sommersitz in Comologno verbrachten Jahre, zwischen 1929 und etwa dem Kriegsende; in Rückblicken wird das frühere Leben der Schriftstellerin erzählt. Dabei erscheinen zum einen das ambivalente, von der Ausübung psychischer Gewalt überschattete Verhältnis zu der Mutter ${ }^{1}$ und zum anderen die Bekanntschaft mit Carl Gustav Jung und seinem psychoanalytischen Kreis sowie enge Kontakte mit den Dadaisten in Zürich (etwa mit Hugo Ball, Hans Arp, Sophie Taeuber) als besonders prägende Erfahrungen. Signifikant war auch die Tätigkeit als Gastgeberin eines Künstlersalons im Baumwollhof in Zürich, dem Wohnsitz Valangins und ihres ersten Mannes,

\footnotetext{
1 Vgl. dazu Kamber 1990, S. 33-52.
} 
des erfolgreichen Rechtsanwalts Wladimir Rosenbaum, in dem u. a. James Joyce, Joseph Roth und Robert Musil zu Gast waren. In dieser Zeit entwickelte sich Aline Valangin zu einer kunstinteressierten, selbstbewussten und modernen Frau, die eine offene Ehe führte und sich neuen Herausforderungen freudig und mutig stellte, bis sie etwa Mitte der 1930er Jahre ihre neue Berufung als Schriftstellerin entdeckt hat.

Im Hinblick auf die anvisierte Fragestellung ist festzuhalten, dass sich der biografische Roman an der Perspektive der Zuwanderer orientiert. Valangin selbst lässt in ihren Romanen (Die Bargada, 1943 / Dorf an der Grenze, 1982) und Erzählungen (Tessiner Erzählungen, 2018) das Leben des engen Tessiner Bergtales in den 1930er und 1940er Jahren in seinem tristen Alltag auferstehen und bevorzugt dabei die Perspektive der Einheimischen. Ihre Geschichten erzählen aber auch von Außenseitern unterschiedlicher Art, Partisanen, Schmugglern und Flüchtlingen und lassen so das Bild der Tessiner Berge als eines Zufluchtsortes für verschiedenste Menschengruppen entstehen.

\section{Das Tal und die Künstler}

Eveline Haslers Werk über Aline Valangin erweist sich von Anfang an als ausgesprochen ortsorientiert und lenkt die Aufmerksamkeit des Lesers mehrfach auf den Schauplatz des Geschehens. Heraufbeschworen wird dabei die spezifische Lage des Onsernone, das gleichsam eine besondere Stelle im geografischen Raum einnimmt:

Der Vater hat es Luca auf der Landkarte gezeigt: „Die Dörfer im Onsernonetal sind gewissermaßen der Scheitel der Welt. Stell dir einen Dachfirst vor, Luca, von dem das Wasser abfließt nach Norden und Süden. Hinter Spruga kann man auf einem Waldweg nach Italien spazieren, und wer gut zu Fuß ist, gelangt über Domodossola westwärts nach Frankreich. Auf der anderen Seite führt der Weg über den Gotthard in die Svizzera interna, von dort aus den Rhein entlang, nach Österreich und Deutschland.“2

Es ist eine interessante, mehrdeutige Vorstellung: Einerseits handelt es sich doch um eine (politische) Grenze zwischen verschiedenen Ländern, andererseits evoziert das besagte Bild die Freiheit und den (inneren) Frieden sowie eine ungeheure Vielfalt von Möglichkeiten, die man ergreifen kann. Der sich dem Blick darbietende, physisch entgrenzte Raum wird auch spirituell definiert. Er lädt geradezu zur Hinterfragung der eigenen Verortung ein. Das so literarisierte Onsernone wird zu einer Schnittstelle von Realem und Fiktivem und präsentiert

\footnotetext{
${ }^{2}$ Hasler 2004, S. 102.
} 
sich dem Leser als ein Ort des Übergangs zwischen Lebenswelt und Kunst - eine Position, die auch Valangins Originalwerk konstituiert. ${ }^{3}$

Durch die Begegnung mit diesem besonderen Standort entsteht die Möglichkeit, seine Identität neu zu definieren resp. zu erweitern. Vornehmlich für die Künstler ist es ein durchaus verlockendes Angebot. Dies wird an der Gestalt des italienischen Schriftstellers und Valangins Geliebten, Ignazio Silone, veranschaulicht und an den Fragen der Toleranz und des Friedens überprüft.

Aline Valangin kam mit den Ideen der Friedensbewegung relativ früh in Berührung, da sich ihr Großvater väterlicherseits, Elie Ducommun, der Friedensnobelpreisträger 1902, aktiv für die Friedensidee engagiert hat. Seinem Vermächtnis ist sie ihr Leben lang treu geblieben, indem sie bereits während ihrer Zürcher Jahre die Emigranten beherbergt und tatkräftig unterstützt hat. Auch in Comologno blieb ihr Haus allen Hilfsbedürftigen offen und ihr Einsatz wurde von ihren Freunden bereitwillig anerkannt. Es ist ihr nämlich gelungen, ihnen allen einen zur Entfaltung notwendigen Raum zu erschaffen, in dem eingrenzende Konventionen aufgehoben und eine tiefere Einsicht ins Wesentliche ermöglicht wurde:

Dann sagte er versöhnlich: „Paolo, ich kann deine Wut nachfühlen, aber von dieser Frau, die wir - zu unserem Glück oder Unglück - offensichtlich beide lieben, habe ich eine Menge gelernt über das Leben, die Menschen und auch über das Klima, in dem wahre Menschlichkeit gedeiht. Ihre Toleranz, Rossi, ist mir ein Vorbild, und die Barca, als Arche für die verschiedenartigsten Menschen - ist sie nicht ein Modell für dieses föderative Europa, das deinem Bruder Ernesto vorschwebt?4

Die Einheimischen in Comologno betrachteten den neuen Zugang in ihrem Dorf zunächst mit Verwunderung und Erstaunen. Den weitgehend armen, durch schwere Arbeit abgehärteten Menschen erschienen die Städter als Vertreter einer anderen Welt: „Der Palazzo ist ein Fremdkörper im Dorf. Die hinter der Mauer haben ihre eigenen Gesetze. “5 Man schreckte aber von den Kontakten nicht zurück, man arbeitete gern für die Zuwanderer, sie wurden gar als eine willkommene Geldquelle genutzt, da man als selbstverständlich annahm, dass sie alle reich sind.

Die Interaktionen zwischen dem Dorf und den Künstlern waren nicht frei von skandalösen Momenten. Die erotische Freizügigkeit der Künstler war für die Bauern im Dorf ungewöhnlich und schwer zu verarbeiten. Das moderne Weiblichkeitsbild, „[...] ein neues Geschöpf [...] frei, geistreich, klug, stolz, von keinem Schuldgefühl belastet oder überschattet" ${ }^{" 6}$, widersprach ihren tradierten Erwartungen und Vorstellungen. Dennoch wurde Valangin wegen ihrer erotischen Unabhängigkeit weder verfemt noch von der Dorfgemeinschaft eindeutig verurteilt. Es lag wohl am Bonus ihrer Position als Außenseiterin, der man

\footnotetext{
3 Zum literaturtopographischen Zugang zu Räumen vgl. Piatti 2008, S. 289.

${ }^{4}$ Hasler 2004, S. 153-154.

5 Ebd., S. 100.

${ }^{6}$ Ebd., S. 166.
} 
paradoxerweise eben deshalb mehr Freiheiten zuerkannt hat. Hasler vermittelt in ihrem Werk den Eindruck, als würden die Dorfbewohner die Künstler vor allem als die Anderen und nicht direkt als die Fremden betrachten. Es ist ein interessanter Ansatz: Das Andere verlangt nämlich weder eine direkte Stellungnahme noch einen Alternativentwurf; es birgt ferner kein Konfliktpotenzial in sich. Es ist eine Kategorie, die eine gewisse Sicherheit gewährleistet. Auf der anderen Seite vermeidet man aber dadurch die Auseinandersetzung mit der Frage nach der Fremdheit im Anderen und entgeht so der Notwendigkeit, hierzu eigene Stellung zu bestimmen. ${ }^{7}$

Für die meisten Besucher bleibt das Tal ein Übergangsort, früher oder später verlassen sie Comologno und suchen ihr Glück woanders. Für Valangin aber erweist sich der Aufenthalt hier, genauso wie ihr Austausch mit den Dorfbewohnern und das Bergerlebnis, als eine Art Grenzerfahrung, die ihr Leben in neue Bahnen gelenkt hat. Denn eben hier findet sie zu einer neuen Lebensaufgabe, indem sie zu schreiben beginnt:

Doch Aline gehört nicht zu jenen Musen, die sich auslöschen ließen von den Genies, die sie inspirierte. Sie hat zwar Kreativität in andern geweckt, dabei aber an ihrer Autonomie festgehalten, es auch verstanden, sich Raum und Kraft zu bewahren für die eigene Begabung. Wie Silone beginnt sie nun Wörter zu bewohnen, ihre neue Liebe gilt dem Schreiben. Sie schreibt mit der gleichen kühlen Leidenschaft, mit der sie am Klavier ihre Fugen spielte. Anstatt mit Tönen, baut sie mit Wörtern ein Thema auf. Dorfgeschichten, gewiss; doch im Wassertropfen spiegelt sich die Welt. ${ }^{8}$

Das Onsernone mutiert so für Valangin zu einem autobiografischen $\mathrm{Ort}^{9}$, der im Kontext der individuellen, persönlichen Entwicklung anzusiedeln ist. Ihre eigenen literarischen Entwürfe werden dann reziprok die symbolische Kulturlandschaft dieses Raumes mitgestalten.

\section{Das Tal und sein Denkmal im Wort}

Die Neuauflage Tessiner Erzählungen (2018) umfasst Valangins Werke, die in den späten 1930er Jahren in zwei Bänden erschienen sind: Geschichten vom Tal. Neun Geschichten aus dem Onsernone (1937) und Tessiner Novellen. Sechs Geschichten aus dem Onsernone (1939). Die Titel beider Sammlungen scheinen ein Beleg für die Absicht der Autorin zu sein, die Eigenart dieses Tessiner Tales für die nachfolgenden Generationen festzuhalten und es so dem kollektiven Gedächtnis der Schweizer einzuverleiben. Der Zusammenhang zwischen dem Raum

\footnotetext{
7 Vgl. dazu Chołuj 2004, S. 283.

${ }^{8}$ Hasler 2004, S. 225.

9 Zum Begriff des autobiografischen Ortes vgl. Czermińska 2011, S. 183-200.
} 
und dem Menschen und insbesondere der Einfluss der rauen Berglandschaft auf die Herausbildung eines besonderen Menschenschlages stehen im Mittelpunkt ihres Interesses. Daran schließen sich die Überlegungen zum Heimatverständnis der Talbewohner an.

In ihren Geschichten zeichnet die Schriftstellerin daraufhin das nicht einfache Leben in Onsernone. Dieses wird weitgehend durch die karge Landesbeschaffenheit bestimmt, die sowohl den Ackerbau als auch die Viehzucht erschwert. Deswegen leiden die Menschen unter der Armut, die die Saisonarbeit der Männer in den außerhalb des Tales liegenden Städten erzwingt. Die regelmäßige Abwesenheit der Männer hat zur Folge, dass die Frauen im Tal besonders selbständig und selbstbewusst sind. Mutig und stolz tragen sie die Verantwortung für den Haushalt und die Familie. Dadurch erringen sie sich auch das Mitspracherecht in den Zeiten, wenn die Männer zu Hause sind. Es verwundert also nicht, dass eben diese recht ungewöhnlichen Frauen die Protagonistinnen vieler Erzählungen sind.

Aufgrund dieser außergewöhnlichen Situation gehören also das Tal und die Tessiner Berge hauptsächlich den Frauen, die größtenteils auf sich selbst gestellt sind und ihr Leben auch unter schwierigen Umständen bewältigen. Ihr Machtanspruch gründet eben auf dieser Stärke und dem Durchstehvermögen. Seit Generationen pflegen sie einige zuweilen seltsame Bräuche, auf die sie aber besonders stolz sind, da sie ihre Ausnahmestellung unter anderen Frauen nachweisen:

Hier waschen die Frauen ihre Wäsche. Sie haben eine seltsame Art zu waschen. Sie waschen nicht mit den Händen, wie das die Frauen der ganzen Welt tun, sie waschen mit den Füßen, indem sie, stehend, ein Zipfelchen des nassen Stoffes mit der großen Zehe packen und damit auf den übrigen, glatt auf einem Stein ausgebreiteten Stoff schlagen. Sie tun es rasch und sicher, mit kleinen Sprüngen, die Hände in die Hüften gestemmt, den Rücken gebeugt. Von weitem sehen die Frauen aus wie Bachstelzen oder hüpfende Sperlinge. Im ganzen Tal weiß man, dass die Frauen des obersten Dorfes auf diese seltsame Art waschen. Man lacht sie deswegen aus, doch im Geheimen werden sie darob bewundert. Denn niemand kann es ihnen nachmachen. Es ist eine Kunst. Und die Frauen selbst tragen im Geheimen die Gewissheit mit sich herum, dass sie dieser Kunst wegen mehr wert sind als alle anderen Frauen des Tales, ja vielleicht als alle Frauen der Welt. Wer weiß? ${ }^{10}$

Selbstverständlich gibt es unter den Frauen fleißige und faule, kluge und dumme, ruhige und streitsüchtige. Sie verkörpern auch die Vorurteile der Einheimischen allen Neuerungen gegenüber. Dies zeigt sich etwa an dem Versuch der Sciora (ein alter ego der Autorin), den Frauen die längst vergessene Kunst des Spinnens beizubringen, damit sie in den Wintermonaten ihr Haushaltsgeld durch die Hausarbeit aufbessern können. Bald beginnen die Eifersüchteleien und Boshaftigkeiten, wodurch das ganze Projekt scheitert. In einer anderen Erzählung

10 Valangin 2018, Elektrizität, S. 128-129. 
sollen die Frauen auf Anordnung der Behörden die Fertigkeit des Strohflechtens wieder erlernen, damit sie Teile der staatlichen Gedenkmedaillen anfertigen können. Die Dorfbewohnerinnen beanstanden die Wahl ihrer Leiterin und wollen nicht auf sie hören. Dabei führen sie recht kuriose Argumente an:

Also Pia. Sie war keine Hiesige. Ihr Vater war aus dem Italienischen gekommen, man erinnerte sich noch gut, dass er vor dreißig Jahren als Holzfäller - damals war er jung und hübsch gewesen - auftauchte und allen Mädchen den Kopf verdrehte. Die Luisa hatte ihn dann geheiratet. Obschon er nicht anders gelebt hatte als die andern Männer im Tal, war er in den Augen aller etwas Besonderes geblieben. Mochten es die nicht ganz vergessenen Erfolge bei den Frauen sein, oder seine klarere Sprache, sein Fleiß im Sparen, oder vielleicht die Art, wie er sich von allen Dorfhändeln zurückhielt: Er wuchs nicht mit dem Dorf zusammen, er blieb der Fremde, und sogar seinen Kindern, die doch hier geboren und aufgewachsen waren, haftete das Besondere an. ${ }^{11}$

Im Grunde genommen geht es um persönliche Animositäten, alte und neue Familienfehden, Parteizugehörigkeiten und nicht wirklich um die Frage der Fremdheit, diese wird vor allem als ein willkommener Vorwand gebraucht, wenn Unangenehmes zur Debatte kommt.

Allerdings gibt es keinen rechten Zusammenhalt zwischen den Frauen, keine weibliche Solidarität. Die Dorfbewohnerinnen sind durchaus nicht frei von Launenhaftigkeit, Streitsucht, Eitelkeit oder Einfalt. Das Leben im Tal ist keine Idylle und manch eine Frau muss unverschuldeterweise gegen die Vorurteile und den Aberglauben ankämpfen, die eben von anderen Frauen angetrieben werden:

Es war Claretta, ihr Patenkind, die Tochter jener einsamen Frau, die zwischen den letzten Tobeln in einem verfallenen Hause mit dem Kind wohnte und von welcher die Rede ging, sie sei eine Hexe. Warum sie eine Hexe sei und was ihr als Hexe zur Last gelegt wurde, war nicht zu erfahren. Sie war eben eine Hexe, und daran war nicht zu rütteln. Niemand wollte mit ihr zu tun haben, es seien dann etwa Männer, und auch die nun im Geheimen ... es galt als Unehre, in der Nähe ihres Hauses zu später Stunde angetroffen zu werden ... ${ }^{12}$

Die Tochter der angeblichen Hexe wird in der Schule gehänselt und misshandelt, trotzdem kann sie auf keine Unterstützung der Erwachsenen zählen. Sie und ihre Mutter werden von der Dorfgemeinschaft in das Außenseiterdasein gedrängt, sie bewegen sich am Rande der Gesellschaft und verbringen die meiste Zeit in ihrem Anwesen in den Bergen. ${ }^{13}$

11 Das Kreuz, ebd., S. 225-226.

12 Versöhnung, ebd., S. 300-301.

13 Diese Familie steht im Mittelpunkt der Werke Die Bargada und Dorf an der Grenze. 
Eine besondere Stellung kommt in der Dorfgemeinschaft den Behinderten als den Anderen zu. Sie gehören zwar zur Gemeinde, weil es als verpönt gilt, Familienmitglieder in eine Anstalt abzuschieben, wo man noch selbst für ihren Unterhalt aufkommen muss, ihre Position ist aber eine schwierige. Der Dorftrottel Carlo wird als Nachkomme einer eingesessenen, aber zu Trinkern heruntergekommenen Dorffamilie zwar geduldet, jedoch mehrfach als der Andere stigmatisiert. Er verrichtet verschiedene Botengänge im Dorf und sichert sich so seine Lebensgrundlage. Wegen seines unberechenbaren Verhaltens, seiner unzusammenhängenden Äußerungen, sowie der ihm zugeschriebenen Gabe, das Wetter vorhersagen zu können, wird er von den anderen Dorfbewohnern als suspekt marginalisiert und in die andere Sphäre des Chaos abgeschoben. ${ }^{14}$ Selbst in den Augen der ihm gegenüber unvoreingenommenen und weltgewandten Sciora scheint er als eine Art rätselhaftes Medium zwischen den Welten zu fungieren - etwa zwischen Natur und Kultur oder zwischen Wirklichkeit und (Alp)Traum:

Dieses Gesicht! Was war das überhaupt ein Gesicht! Eher war es eine Landschaft mit Buckeln und Bergen, wilden Hängen, Schluchten und Schründen, verwüsteten Wäldern, Felsblöcken, Strudeln in Löchern, Äckern nach Erdbeben, Lawinenzügen und Bergstürzen. Aus der Urwelt dieses Gesichtes schauten zwei Augen, das eine klein und matt, vom entzündeten Augenlid fast verdeckt. Doch aus dem anderen kam ein Strahl der Vernunft aus Gottes Himmel auf die Wüstenei der Erde. ${ }^{15}$

Tatsächlich verfügt Carlo über eine besondere musikalische Sensibilität und ahmt mehr oder weniger bewusst bestimmte Töne nach. Seine Zuflucht in der Welt voller Unverständnis und Unsicherheit findet er eben in seiner Musik, wo er eine Art unio mystica erlebt. Die fremde Sciora, eine aufmerksame Beobachterin, hört ihm als einzige zu, geht auf ihn ein und ehrt seine Eigenart. Auch wenn ihm gewöhnliche menschliche Eigenschaften abgehen, befindet er sich aufgrund seiner Mängel in einer Ausnahmeposition, indem er an der Sphäre des sacrum partizipiert. ${ }^{16}$ Freilich kann es nur eine Außenstehende, eine begnadete Musikerin und in diesem Sinne eine Seelenverwandte erkennen.

\section{Das Tal und der Krieg}

Im Roman Dorf an der Grenze, der wegen der dezidierten Kritik der Autorin an der Schweizer Politik während des Zweiten Weltkrieges erst 1982 erscheinen konnte, reflektiert Aline Valangin ausführlich über die Kriegsjahre im Onsernone. Die Tessiner Berge - für die Einheimischen bisher ein recht überschaubarer und

14 Vgl. Moszyński 1967, S. 97.

15 Valangin 2018, Carlo, der Narr, S. 95.

16 Vgl. Moszyński 1967, S. 413. 
vertrauter Raum - mutieren hier zu einem Ort der Begegnung mit der Gewalt und mit dem Fremden und zum Prüfstein der eigenen Menschlichkeit.

Das Leben im Tal läuft seit Jahrzehnten den gewohnten Gang, der sich nach den Jahreszeiten und der saison- und arbeitsmarktbedingten Abwesenheit der Männer richtet. Nichts verändert sich und alle nehmen es als selbstverständlich hin: „Dem Dorf schlägt keine Stunde. Seit Jahren geht die Kirchenuhr nicht mehr. Ihre Zeiger sind gebrochen. Nutzlos liegt der Kranz der Ziffern um die leere Scheibe. Die Zeit steht hier still."17 Der Krieg kommt den Dorfbewohnern am Anfang wie eine Naturerscheinung vor, die sie selbst gar nicht betrifft, und doch macht er ihre heimatlichen Berge zum Schauplatz der gewalttätigen Auseinandersetzung und Begegnung mit dem Ungewohnten. Die Veränderungen scheinen zunächst nicht nur negativ zu sein: Die Männer werden zwar einberufen, man bekommt aber Geldzuwendungen vom Staat. Bald werden jedoch im Gegenzug erhöhte Abgaben an Lebensmitteln verlangt, was wegen kargen Dorfbodens nur schwer zu bewältigen ist. Die verschlafene Berggrenze gewinnt unversehens an Bedeutung und wird mit verstärkten Kontingenten italienischer und schweizerischer Grenzwächter besetzt. Unvermittelt und unerwartet werden die Dörfler mit den Flüchtlingen konfrontiert, die über die Grenze kommen. Trotz ihres üblichen Misstrauens den Fremden gegenüber werden die Flüchtlinge im Dorf spontan willkommen geheißen, insbesondere Frauen und Kinder erwecken sogleich das Mitleid aller. Man möchte ihnen Zuflucht gewähren und begegnet daher der Staatsanordnung, alle Flüchtlinge zurückzuschicken, mit Unverständnis und Empörung. Man fühlt mit den Menschen, die alles aufgeben mussten, mit und wird sich durch die Konfrontation mit ihrem Schicksal der Fragilität der eigenen Existenz bewusst: „Wie seltsam: Es genügte also nicht, Hab und Gut zu opfern und mit Anspannung der letzten Kraft als Bettler über die Grenze zu fliehen? Es wurde mehr verlangt. Was denn?"18 Man empört sich gegen die Schlepper, die den Juden skrupellos ungeheure Geldsummen für die Führerdienste über die Grenze abnehmen. Die Situation spitzt sich allmählich zu und der Menschenstrom kommt einer Naturgewalt gleich:

Die zwei jüdischen Familien hatten den Anfang gemacht. Sie waren Vorboten des Flüchtlingsstromes gewesen, der sich nun von den Bergen herunter ergoß. Sie drangen einzeln und in Gruppen ein, ausgestattet mit viel Gepäck oder abgerissen in Lumpen, halb erfroren und verhungert, voller Wunden, unfähig, sich zu erklären, irrsinnig. An der Grenze wußte sich die verstärkte Wache kaum zu helfen. „Die Leute purzeln von den Bergen wie Wasserfälle, wenn's regnet“, klagte Bozi, „Sie rinnen einem zwischen den Fingern durch. ${ }^{\text {19 }}$

Im Laufe der Zeit kommen nicht nur die Juden, sondern die Menschen aus aller Welt über die Grenze. Die Dorfbewohner solidarisieren sich ausnahmslos

17 Valangin 2002, S. 171.

18 Ebd., S. 207.

19 Ebd. 
mit allen und lehnen sich gegen die undurchsichtigen staatlichen Regeln auf, die über die Aufnahme oder die Abschiebung entscheiden.

Aber auch andere Menschengruppen nehmen nun die heimatlichen Berge um das Dorf herum in Beschlag. Wenn nach einiger Zeit die Vorräte knapp werden, kommen die Schmuggler über die Grenze und versorgen die Dorfbewohner mit Lebensmitteln und Luxusgütern. Sie nehmen ein großes Risiko auf sich, denn sie müssen unwirtliche und gefährliche Stellen überwinden. Da sie sich aber in der Gegend gut auskennen, werden die Berge für sie zu Verbündeten; trotz zahlreicher Gefahren gewähren sie paradoxerweise zugleich Schutz und Sicherheit:

Die Weiden dehnten sich breit und flach einige Stunden weit um die Hütte aus. Mehrere Spuren wiesen in ein schmales Tälchen, in dem zwischen Bergrücken ein kleiner See lag, eher ein Tümpel, mit klarem, kalten Wasser, dessen dunkler Grund es schwarz erscheinen ließ. Die wenigen Lärchen, die hier noch standen, waren alle vom Blitz getroffen. Bis hierher wuchsen Heidelbeerstauden und Alpenrosen. Weiter aufwärts war alles kahl und öde. Einzelne Vögel huschten herum. Sonst nichts Lebendiges. Das kurze Gras hier war früh verbrannt und grünte spät im Frühling. Wenig höher oben verlief die Grenze auf der Paßhöhe. Der Blick konnte dort weit in die Runde schweifen bis zu entlegenen Höckern, die schroff und abweisend für die Dörfler keine Namen mehr trugen. In dieser wilden, unwirtlichen, felsigen Gegend voller Geröllhalden und böser Abstürze, suchten sich die Schmuggler ihren Weg. Er war gefährlich und voller Tücken, doch lauerte hier kein Grenzwächter, kein Soldat, kein Spürhund. Sie gingen sicher. ${ }^{20}$

Die Einstellung der Dörfler den Schmugglerbanden gegenüber ist ambivalent. Die Rechtschaffenen empören sich gegen die Gesetzesbrecher, die anderen sind froh, dass sie die begehrten Güter erwerben können. Das Schmugglergeschäft floriert mit dem stillschweigenden Einverständnis des Dorfes, denn auch die Unzufriedenen halten still. Und auch wenn die Schmuggler mit der Zeit die Preise für die Lebensmittel erhöhen und sich auf gewinnbringende Luxusgüter einstellen, werden sie nicht denunziert.

Unter die Schmuggler mischen sich im Stillen politisch engagierte Spione, die möglichst viele Bergübergänge und -routen für die Widerstandsbewegung auskundschaften. Auch diese Gruppen finden nun in den Bergen Unterschlupf und nehmen sie in ihren Besitz. In der Abgeschiedenheit des Gebirges errichten sie ihre Ausbildungslager und bereiten sich auf ihre Attentate vor:

Die Partisanen, die man im Dorf beinahe vergessen hatte, hatten sich in Banden gesammelt, unter eigenen Anführern. Sie waren mit Waffen versehen und ausgebildet worden, und der Wille, ihr Land von der Plage der Bedrücker zu befreien, trieb sie an. Sie stürzten aus ihren Schlupf-

20 Ebd., S. 220. 
winkeln in den Bergen auf den Feind, lieferten ihm Gefechte, sprengten Brücken, um ihn abzuschneiden, was ihnen so gut gelang, dass sie ein Dorf nach dem anderen in ihre Hand bekamen, und die Deutschen sich in den letzten Winkel der Berggegend gedrängt sahen. Eine Frage der Zeit, wann sie sich ergeben mußten. ${ }^{21}$

Da die politischen Ansichten der Dörfler unterschiedlich sind, bekommen die Partisanen zunächst keine uneingeschränkte Unterstützung, sie werden jedoch geduldet und den Behörden nicht angezeigt. Am Ende kommt es jedoch zu einer blutigen Auseinandersetzung an der Grenze, an der sich außer den Faschisten, Grenzschützern, Partisanen und Flüchtlingen auch die Dörfler beteiligen und sogar Todesopfer zu beklagen haben. Durch diese traumatische Erfahrung aufgerüttelt, sind sie danach alle derselben Meinung, dass man sich nämlich für die richtige Sache auch gegen die behördlichen Anordnungen aktiv einsetzen sollte. Die Solidarität sollte alle Grenzen überwinden: „Menschen, die sich für die Freiheit schlagen, sind an jener Stelle, auf die es ankommt, gar nicht geschätzt.“22 Man darf sich demzufolge auch nicht der Pflicht entziehen, für seine Überzeugungen und für die gemeinsame Sache zu kämpfen: „Jetzt erst begreifen wir, daß wir nicht, wie unsere Turmuhr, ohne Zeiger stehenbleiben können [...]. Wir müssen mitmachen ... wir müssen unsern Sinn der Zeit öffnen und Anteil nehmen am Geschick der Freiheit ... die auch unsere Freiheit ist.“23

\section{Fazit}

Alles in allem sollte festgehalten werden, dass das Onsernonetal der 1930er und 1940er Jahre in den gesichteten Werken als ein Ort erscheint, der überraschend offen ist und Menschen unterschiedlichster Provenienz Zuflucht gewährt. Das Tal bleibt zwar nicht frei von (latenten) Spannungen, aber im Grunde genommen lässt es doch alle willkommen heißen und bietet sich als Katalysator für die Selbstfindung oder Prüfstand für die Menschlichkeit an.

Eveline Haslers Roman ist ein Beispiel für eine moderne fiktionale Dichterbiografie, die sich durch eine multiperspektivische Auffächerung des erzählten Geschehens auszeichnet (u.a. auktoriale Erzählinstanz, Tagebuchauszüge, Figurendialoge). ${ }^{24}$ Wie bereits gezeigt wurde, funktionalisiert die Autorin auch die Raumdarstellung als einen wichtigen Bedeutungsträger. Interessant erscheint in diesem Kontext die Frage nach dem Einfluss moderner Theorien auf die angebotene Darstellungsweise. Gemeint wird hier z.B. die Begünstigung der Kategorie des Anderen gegenüber der des Fremden in der Sicht der Talbewohner auf die Künstler. Valangin selbst entzieht sich in ihren Texten weitgehend dieser Frage,

${ }^{21}$ Ebd., S. 264.

22 Ebd., S. 332.

23 Ebd., S. 333.

24 Vgl. dazu Nünning 2000, S. 28. 
indem sie sich vorwiegend auf die Interaktionen unter den Dorfbewohnern selbst konzentriert (auch wenn das alter ego der Autorin, die Sciora, in den Geschichten auftaucht, nimmt sie weitgehend die Beobachterposition ein) und ihre zuweilen zweifelhaften Motivationen schonungslos entlarvt.

Zweifelsohne erweist sich der geopoetische Zugang zu Haslers Text als besonders inspirierend, weil hier der Beziehung zwischen dem Subjekt und dem geografischen Raum im Hinblick auf die dichterische Kreation der (Auto)Biografie Valangins eine besondere Bedeutung innewohnt. Mit der Beschreibung des Palazzo La Barca in Comologno fängt der Roman an und schafft so einen signifikanten Rahmen für das (nach)erzählte Leben. Der geografische Raum fungiert dabei keineswegs nur als eine einfache Kulisse, sondern vielmehr als ein Bereich diverser Erfahrungen, ${ }^{25}$ die einerseits Valangins Leben und ihre intellektuelle Entwicklung beeinflussen und andererseits das landschaftliche und kulturelle Spezifikum des Onsernone festhalten und mitgestalten.

Valangins Roman Dorf an der Grenze reflektiert nicht nur über den Krieg, sondern auch über die Erfahrung der Grenze, die sich überraschenderweise zugleich als eine Grenzerfahrung entpuppt, und untersucht, was das Wissen über die Landesgrenze eigentlich bedeutet - ein Problem, dessen sich die Einheimischen bis zum Krieg überhaupt nicht bewusst waren, da sie die Grenze vor ihrer Haustür als eine gegebene und vertraute Tatsache angenommen haben, aus der keine besonderen Konsequenzen entstehen. Nun wird sie zum Prüfstand ihrer eigenen Menschlichkeit; durch die Erfahrung von Gewalt, Leid und Tod entsteht ein quasi „,körperhaftes Bewusstsein der Grenze“26. Infolgedessen partizipiert Aline Valangin an der Diskussion über das Wesen des Schweizer Patriotismus nicht nur mit ihrer dezidierten Kritik der Schweizer Emigrantenpolitik während des Zweiten Weltkrieges, sondern auch mit ihrem Entwurf der Grenze als mannigfache Grenzerfahrung.

\section{Literaturverzeichnis}

\section{Primärliteratur}

Hasler, Eveline (2004): Aline und die Erfindung der Liebe, 2. Aufl., München: dtv. Valangin, Aline (2002): Die Bargada / Dorf an der Grenze. Eine Chronik, Zürich: Limmat. Valangin, Aline (2018): Tessiner Erzählungen, Zürich: Limmat.

\section{Sekundärliteratur}

Chołuj, Bożena (2004): Inność w powieściach Teodora Fontane, in: Maria Janion / Claudia Snochowska-Gonzales / Kazimiera Szczuka: Inny, inna, inne. O inności w kulturze, Warszawa: IBL, S. 283-288.

25 Vgl. Rybicka 2014, S. 280.

26 Matt 2004, S. 115. 
Czermińska, Małgorzata (2011): Miejsca autobiograficzne. Propozycja w ramach geopoetyki, in: Teksty Drugie, H. 5, S. 183-200.

Kamber, Peter (1990): Geschichte zweier Leben. Wladimir Rosenbaum \& Aline Valangin, 3. Aufl., Zürich: Limmat.

Matt, Peter von (2004): Der Traum an der Grenze. Zur literarischen Phantasie in der Schweiz, in: ders.: Die tintenblauen Eidgenossen. Über die literarische und politische Schweiz, München: dtv, S. 113-122.

Moszyński, Kazimierz (1967): Kultura ludowa Stowian, Bd. 2, Teil 1, Kultura duchowa, 2. Aufl., Warszawa: Książka i Wiedza.

Nünning, Ansgar (2000): Von der fiktionalen Biographie zur biographischen Metafiktion. Prolegomena zu einer Theorie, Typologie und Funktionsgeschichte eines hybriden Genres, in: Christian von Zimmermann (Hrsg.): Fakten und Fiktionen. Strategien fiktionalbiographischer Dichterdarstellungen in Roman, Drama und Film seit 1970, Tübingen: gnv, S. 15-36.

Piatti, Barbara (2008): Die Geographie der Literatur. Schauplätze, Handlungsräume, Raumphantasien, Göttingen: Wallstein.

Rybicka, Elżbieta (2014): Geopoetyka. Przestrzeń i miejsce we wspótczesnych teoriach i praktykach literackich. Kraków: Universitas. 